\title{
Pediculosis palpebrarum initially diagnosed as blepharitis
}

\author{
Ebeigbe JA* and Osaiyuwu AB
}

Department of Optometry, Faculty of Life Sciences, University of Benin, P.M.B 1154

Benin City, Edo State, Nigeria

<petedidi2000@yahoo.co.uk>

\begin{abstract}
Pediculosis is an infestation of lice. Eyelid involvement is uncommon, but can be caused by pediculus humanus capitis (head louse), rarely by pediculus humanus corporis (body louse) and commonly by phthirus pubis (pubic louse). Signs and symptoms are similar to those of blepharitis. A diagnosis of any type of pediculosis requires the finding of live specimen of lice and/or a viable nit.
\end{abstract}

The immediate and effective treatment of pediculosis palpebrarum is removal of the lice and nits manually with forceps and treating the patient with vaseline, twice daily for seven to ten days. Also, fomites such as pillow cases, bedsheets, towels and hats need to be washed in hot water and dried. This is to avoid further spread of lice or reinfestation.

Key words: lice, infestation, pediculosis, madarosis.
Pediculosis palpebrarum is an infestation of the eyelids by pediculus humanus capitis (head lice). Pediculus species are 2-4 mm long and typically infest the hair ${ }^{1}$. Infestation of the cilia however, is rare. The common cause of eyelid infestation is phthiriasis pubis (pubic louse) $)^{2-5}$. Ocular signs and symptoms include bilateral ocular itching, irritation, visible lice and nits, erythematous lesions resulting from louse bites, reddish brown deposits on the lashes (louse faeces), secondary blepharitis, follicular conjunctivitis and marginal keratitis. Head lice are spread through direct head to head contact with an infected per$\operatorname{son}^{6-9}$. Factors that predispose to head louse infestation include young age, close crowded living conditions, warm weather and sex. Females are said to be at higher risk of headlice infestation than males, because of social behaviour like close physical contact (hugging, sharing of hats, combs, hair ties) which females are more likely to be involved in. Black persons are possibly less commonly affected by head louse infestation than any other racial group ${ }^{10-12}$. This is partially due to the use of pomades and perhaps because the claw size of the head louse is more adapted to the round shape of the hair shaft found in Caucasians and people of Asian origin ${ }^{10-12}$. Due to similarities in the signs and symptoms of pediculosis palpebrarum and blepharitis, the two conditions can easily be misdiagnosed by clinicians. Diagnosis of any type of pediculosis requires the finding of live specimen of lice and/or a viable nit $^{13-14}$. 


\section{Case report}

A 53-year-old Black female, AB was brought to the Optometry clinic of the University of Benin, on the $1^{\text {st }}$ of November, 2008 by her daughter. Her chief complaints were eye irritation, itchy eyelids and gummy lids in the mornings of one month duration. AB had no formal education and could neither read nor write. The daughter said they had visited two private clinics where her mother was treated for eye infection. The optometrist in the second clinic visited said her mother had blepharitis and placed her on some eye drops and ointment. She said, despite the drugs given, the eyes were not improving. Oculo-visual and her medical history were not remarkable. Her family medical and oculo-visual history were also not significant.

\section{Clinical findings}

Visual acuity measured using the Snellen Tumbling $\mathrm{E}$ chart at 6 meters, revealed unaided presenting VA: OD 6/6 and OS 6/6 . External ocular examination with penlight showed a chalaxion upper lid OS, moderate lid edema and erythema OU, moderate crustations, madarosis, pupils were round, equal and reacted to light. Slightly hyperaemic bulbar and palpebral conjunctiva OU. Extraocular muscles were unrestricted in all positions of gaze. Ophthalmoscopic findings showed a clear lens and clear vitreous. Optic disc was pink with distinct margins OU. Physiologic cupping of 0.3 was noted. Retinal vessels appeared normal with arterio-venous ratio of 2:3. Both maculae were normal with moderately bright fovea reflex.

Slit lamp examination revealed nits and lice attached to the skin and lashes of lid. The nits and lice were removed from the lid with forceps and cotton wool soaked in mentholated spirit was used for debridement. AB was asked if she had any history of pubic lice, as this was the common cause of eyelid infestation. She replied no and said she was no longer sexually active. She however said that she had cut her hair some weeks earlier because she had lice. Microscopic examination subsequently confirmed the lice picked from her lashes as pediculus humanus capitus.

$\mathrm{AB}$ was diagnosed with pediculosis palpebrarum and was told to apply vaseline to the eyelids twice daily for a week. She was counseled on the need for the treatment of close contacts like family members and friends to help prevent further spread of lice and reinfestation. Also, the need for fomites such as pil- low cases, bedsheets, towels and hats to be washed in hot water and dried was stressed. Slitlamp examination performed a week later, revealed clear healthy lids and lashes OU, quiet bulbal and palpebral conjunctiva OU. She was told to stop using the vaseline and reminded of the need for proper eye hygiene and indeed proper hygiene for the whole body. The importance of domestic and environmental hygiene was also stressed.

\section{Discussion}

There are three varieties of blood sucking lice parasites found on man. Out of the three, the body louse is never found in eyelashes. The affliction of eye lashes is rare; but when it occurs, it is usually due to the crab-louse (phthirus pubis) and rarely due to the head-louses (pediculus humanus capitis) ${ }^{15-16}$.

$\mathrm{AB}$ had head lice, which she treated by shaving off her hair and applying kerosene to her scalp. However, she had no history of pubic infestation and was no longer sexually active. This made the possibility of infestation by phthirus pubis very slim, since it is usually transferred through sexual contact. Phthirus pubis is known to be the commonest cause of eyelid infestation ${ }^{15-18}$.

A number of treatment options are available for eyelid infestation by lice. This includes asphyxiants: treating with twice daily application of petrolatum for seven to 10 days. The petrolatum covers the lice and their nits, preventing respiration. The dead lice are then removed mechanically with tweezers or forceps. Alternative treatments include anticholinesterase eye ointments, yellow oxide of mercury, or $20 \%$ flourescein ${ }^{15-19}$. However, the simplest technique for the treatment of eyelid lice is direct removal of the lice and nits with fine forceps, which was the treatment option for $\mathrm{AB}$, and it yielded good result. Cryotherapy is also said to provide a fast cure. Application of $1 \%$ gammabenzene hexachloride cream, permethrin ointment and oral ivermectin are other options. The best line of treatment for $A B$ was with petrolatum. This is because it was the cheapest and most easily available of all the options. Vaseline or petroleum jelly is a common household item. It was also important to counsel $\mathrm{AB}$ about the treatment of family members, friends and / or other close contacts to prevent further spread of lice, and reinfestation. The need for fomites to be washed in hot water and dried is also important in management and prevention ${ }^{20}$. 


\section{References}

1. Di Stefani A, Hofmann-Wellenhof R, Zalaudek I. Dermoscopy for diagnosis and treatment monitoring of pediculosis capitis. J Am Acad Dermatol 2006 54(5) 909-911.

2. Leung AK, Fong JH, Pinto - Rojas A. Pediculosis capitis. $J$ Pediatr Health Care 20056 369- 373.

3. Yoon KS, Gao JR, Lee SH. Permethrin- resistant human head lice, pediculus capitis and their treatment. Arch Dermatol 2003139 994-1000.

4. Heukelbach J, Hermann F. Ectoparasites - The underestimated realm. Lancet 2004363 889-891.

5. Roberts RJ, Burgess IF. New head - lice treatments: Hope or hype? Lancet 20059453 1044-1047.

6. Flinders DC, De Schweinitz P - Pediculosis and scabies. Am Family Phys 200469 341-352.

7. Blenkinsoppi A. Head lice. Pry Health Care 200313 3334.

8. Nash B. Treating head lice. BMJ 2003326 1256-1257

9. Willem S, Lapeere H, Haedens N. The importance of socio- economic status and indinvidual characteristics on the prevalence of head lice in school children. Eur J Dermatol 200515 387-392.

10. Elston DM. Drugs used in the treatment of pediculosis. $J$ Drugs Dermatol 20054 207-211.

11. Burgess IF. Human lice and their control. Annual Review of Entomology 200449 p 457.

12. Zepf B. Treatment of head lice: Therapeutic Options. Am Family Phys 200469 p 655.

13. Meinking TL. Clinical update on resistance and treatment of pediculosis capitis. M J Mad 200410 3264-3268.

14. Pealman DL. A simple treatment for head lice: dry - on, suffocation - based. Pediculicide 2004114 275-279.

15. Nadini M, Priyanka D, Rahul D. Phthiriasis Palpebrarum: An unusual presentation of blepharitis Internet J Tropical Medicine 200521.

16. Turow VD. Phthiriasis palpebrarum: an unusual cause of blepharitis. Arch Pediatr Adolesc Med 1995149704 - 705.

17. Rundle PA, Hughes DS. Phthirus pubis infestation of the eyelids. Br J Ophthalmol 199377815 - 816.

18. Ashkenazi I, Desatnik HR, Abraham FA. Yellow mercuric oxide: a treatment of choice for phthiriasis palpebrarum. $\mathrm{Br}$ J Ophthalmol 199175356 - 358.

19. Lopez GT, Garcia LI, Martinez GJ. Phthiriasis palpebrarum: diagnosis and treatment. Arch Soc Esp Oftamol 2003 78 365-374.

20. Ikeda N, Nomoto H, Hayasaka S, Na-Gaki Y. Phthirus pubis infestation of the eyelashes and scalp hairs in a girl. Pediatr Dermatol 200320 p356. 